\title{
ARM FUNCTION AND MUSCLE ACTIVITY RESPONSES AFTER USING THE KINESIO TAPING INHIBITORY TECHNIQUE
}

original paper

() Wroclaw University of Health and Sport Sciences

DOI: https://doi.org/10.5114/hm.2022.107973

\author{
RODOLFO SILVA KÓS ${ }^{1 \oplus}$, THOMAS W.J. JANSSEN ${ }^{2}{ }^{\circledR}$, ALBERTO CLIQUET JUNIOR $^{1 \oplus}$ \\ ${ }^{1}$ Department of Orthopaedics and Traumatology, State University of Campinas, Campinas, Brazil \\ ${ }^{2}$ Faculty of Behavioural and Movement Sciences, Vrije Universiteit Amsterdam, Amsterdam, the Netherlands
}

\section{ABSTRACT}

Purpose. This study aims at showing the effects in muscle activity and arm function under the influence of the inhibitory muscle technique of the Kinesio tape method in healthy muscles.

Methods. Overall, 20 apparently healthy subjects underwent the procedures 3 times, in 3 different tape conditions: no tape, Kinesio tape, and non-elastic tape. The muscle activity was obtained in millivolts through electromyography on biceps brachii and deltoid during 3 specific tasks, and the arm function was determined in seconds with the Action Research Arm Test, a standardized tool conceived to assess upper extremity function, divided into the subscales of grasp, grip, pinch, and gross movement.

Results. The electromyography results and most of the Action Research Arm Test dimensions did not show any statistically significant differences in any comparison $(p>0.05)$ between the tape conditions. However, there could be a tendency to perform movements faster under the influence of the Kinesio tape, as implied in the dimensions of grip $(p=0.01)$ and total $(p=0.04)$ of the Action Research Arm Test.

Conclusions. The Kinesio tape inhibitory muscle technique and tape conditions did not evoke significant effects in muscle activity or arm function in apparently healthy muscles.

Key words: electromyography, muscle contraction, upper extremity problems, bandages

\section{Introduction}

For reaching and targeting an isolated muscle, the Kinesio taping (KT) method has shown important results regarding muscle facilitation, inhibition, and muscle function in general as an auxiliary tool for rehabilitation. It was developed by Kenzo Kase in 1973 and consists in applying self-adhesive elastic tapes on the skin to provide aid to the muscle and other soft tissues to continually seek homeostasis. In addition to influencing muscle function, the KT method can exert several other physiological effects, which basically depend on the amount of stretching applied to the tape and where and how it is used. The benefits for the patient include joint positioning, improvement of circulation of body fluids, tissue repair, improvement of fascia alignment, sensory stimulation, facilitating or limiting move- ment, increase of proprioception, functional performance enhancement, and lymphoedema control and management [1-3].

The inhibitory muscle technique $[4,5]$ offers enhancement of the muscle relaxation and function, decreasing the muscle activity, and interventions in episodes of fatigue, pain, spasms, muscle injury contractures, and unbalanced muscle activities. It has its application starting from the muscle insertion and finishing around the origin, always in the stretched position of the joint. When the limb returns to a neutral position after the application, the tape becomes wrinkled, and this excess of tape pulls the skin upwards, lifting also the fascia and soft tissues, which causes stretching of the Golgi tendon organ in the distal end of the muscle and suggests muscle relaxation through the reflex peripheral mechanism [2, 3].

Correspondence address: Rodolfo Silva Kós, Laboratory of Biomechanics and Rehabilitation of the Locomotor System,

Vital Brazil Street, 251, Clinics Hospital of the State University of Campinas, $3^{\text {rd }}$ Floor, Cidade Universitária Zeferino Vaz, Campinas, São Paulo, Brazil, e-mail: rodolfo.kos@gmail.com, https://orcid.org/0000-0001-6867-7737

Received: November 5, 2019

Accepted for publication: February 8, 2021

Citation: Kós RS, Janssen TWJ, Cliquet Junior A. Arm function and muscle activity responses after using the Kinesio taping inhibitory technique. Hum Mov. 2022;23(3):65-71; doi: https://doi.org/10.5114/hm.2022.107973. 
The non-elastic (NE) tape is a rigid tape, used mostly for local tension. It was introduced by McConnell in 1984 and has also been demonstrated to improve muscle strength, biomechanical alignment, proprioception, muscle control, and functional movement [6]. Jung et al. [7] affirm that the NE tape prevents the application of excessive force to a specific joint by limiting its function and range of motion. They also hypothesize that there is some influence on muscle activity as joint movement limitation reduces muscle activity.

Kim and Kim [6] compared the KT and NE tape impact on handgrip strength in healthy subjects and found statistically significant differences immediately after KT application, whereas NE brought about no differences when the status before and after the rigid tape application were compared. Nevertheless, there were no differences when the scores were compared between KT and NE, which demonstrates that there were only changes in muscle strength in the group that used KT, but the amount of strength acquired was not sufficient to show any effectiveness when these 2 techniques were compared. Lemos et al. [1] also affirmed in their study that the grip strength increased by the tape was maintained for 48 hours after the tape application.

Regarding the KT inhibitory muscle technique, Huang et al. [8] used the inhibition of plantar flexor muscles during jump activity in able-bodied subjects, assessed by electromyography (EMG). They found an increase in the activation of the medial gastrocnemius immediately after the tape application. However, the height of the jump was not increased. In turn, in the placebo application of KT, there were no changes either in the jump height or in the muscle activity.

Poon et al. [9] suggested that the real action of the tape could be exhibited in a randomized controlled trial using placebo techniques for comparison of results, with no use of tension at the time of application and no rules for initiation and end in the tape application. Cai et al. [3] cited lack of evidence analysing the inhibitory muscle technique, with only the strength assessed and no data related to the EMG activity.

Although the tape application practices are used for several different therapeutic effects, there are few studies that approach specifically EMG activity in the KT inhibitory technique [4]. In addition, there is scarce scientific evidence on KT and most reports are related to muscle facilitation. The KT inhibitory technique needs more investigation regarding its effectiveness to be used in clinical practice in cases of overuse, excessive muscle activity, spasms, and contractures $[1,9,10]$.

This study aims at showing the effects in muscle activity and arm function under the influence of the inhibitory muscle technique of the KT method in healthy muscles. Previous evidence revealed enhanced function compared with NE tape and we therefore hypothesized that the technique would affect both function and activity in healthy muscles.

\section{Material and methods}

\section{Participants}

A total of 20 apparently healthy individuals were recruited to participate in this cross-sectional study based on sampling of convenience. There were 13 males and 7 females, 18 right-handed and 2 left-handed participants. The procedures were performed at the Amsterdam Rehabilitation Research Centre Reade. The volunteers were required to have the entire range of motion for shoulder abduction and flexion. The exclusion criteria involved painful arc of movement, open wound in the area of tape application, allergy to the tape, any kind of neurological affection that would lead to a muscle commitment, failure to perform movements over the shoulder line of the dominant upper limb, and no signed informed consent. The patients received verbal and written explanations of all the procedures before undergoing the interview for characterization of the sample with items related to gender, age, and dominance.

\section{Procedures}

The following procedures were performed 3 times in all volunteers, with 3 different tape conditions: no tape, KT, and NE (microporous tape). The order of the tape conditions was chosen by simple randomization [11-13].

The KT application was performed by a KT practitioner. The Kinesio Tex Gold Fingerprint tape was used, in black colour, and the technique applied within the KT method was the muscle inhibition technique. Both deltoid and biceps tapes had a Y cut, with the muscle belly area apparent between the 2 tape stripes. The deltoid application started in the insertion, in the lateral and medium part of the humerus, with the two Y stripes, containing the therapeutic zone, around the deltoid muscle belly on the anterior and posterior parts of it, and the application finished around the origin. The biceps brachii application started with the anchor on the distal tendon of the biceps, next to the elbow joint, whereas both stripes were placed on the edge of the muscle, finishing with both anchors next to the anterior deltoid. The paper off technique was used, 
with around $10 \%$ of tape stretching, in a stretched position of the limb in all of its area. For the deltoid application on the anterior fibres, the shoulder was in a stretched position with extension and external rotation, stretching the anterior fibres, and the back stripe was also placed on a stretching position, with the shoulder in flexion and internal rotation. For the biceps brachii application, the elbow and the shoulder were kept in extension and pronation, and the 2 stripes of the $\mathrm{Y}$ cut bypassed the muscle belly $[4,5]$.

The NE microporous tape (Heka Por, Van Heek Medical, Venray, the Netherlands) was cut into 2 stripes of $2.5 \mathrm{~cm}$ of width, the same size as KT when cut in half to perform the Y technique. The NE tape application had the same configuration as that with KT, with the two $2.5-\mathrm{cm}$ stripes around the muscle belly, and did not follow any beginning and end protocol or stretched position of the limb - it was only superimposed on the skin. Both applications can be seen in Figure 1.

The muscle activity assessment process was performed through EMG of the deltoid and biceps muscles with a Delsys Trigno Wireless (Trigno Avanti Platform, Delsys Inc., Natick, MA, USA) device. The positioning of the electrodes followed the SENIAM (Surface EMG for Non-Invasive Assessment of Muscles) protocol [14]; a sensor was positioned on the middle deltoid muscle belly, and the other one on the biceps brachii muscle belly. The KT and NE applications left the muscle belly apparent for placing the sensors on it (Figure 1).

The individuals were sitting on a chair with a back rest, with the arms resting on a table at the height of the elbow joint; the subjects started the function from this rest position.
The muscle activity was measured during 3 specific tasks performed with the dominant upper limb: (1) to reach the top of the head with the hand, simulating daily life activities that are supposed to be performed over the shoulder line, such as doing the hair, reaching some high spot, or dressing activities; (2) to move the hand towards the mouth, simulating feeding activities; and (3) to throw a medicine ball of $3 \mathrm{~kg}$, trying to reach high peaks of contraction during the EMG assessment. The tasks can activate both the deltoid and biceps brachii muscles properly, and it is adequate to measure this muscle activity during the performance [15-17]. The tasks were performed 3 times each, and all the measures were used for the final analysis and comparison.

The raw data of the EMG were obtained right after the assessment, and then transferred from the EMG Works Acquisition software (Delsys EMGworks Acquisition 4.7.1, Delsys Inc., Natick, MA, USA) to EMG Works Analysis (Delsys EMGworks Analysis 4.3.1.0, Delsys Inc., Natick, MA, USA), in which the numerical values in millivolts of the EMG activity of both muscles were obtained. These numerical data were transferred to MATLAB (MATLAB R2019a, MathWorks Inc., Natick, MA, USA) for filtering and achieving absolute values. The contraction peaks were obtained manually for the 3 movements within the tasks, and the peak contractions of the muscle were selected manually, which resulted in 3 peak values of the 3 contractions during the trials. If the participant was performing task one, 3 values were obtained from the 3 trials conducted within this task, and then the same values were also obtained for the other muscle.

The Action Research Arm Test (ARAT) is originally used to assess upper limb functions after stroke. It can
A

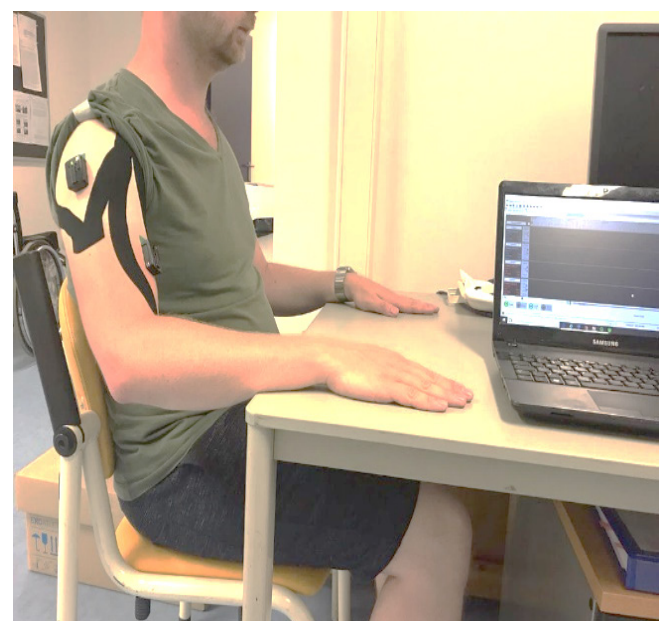

B

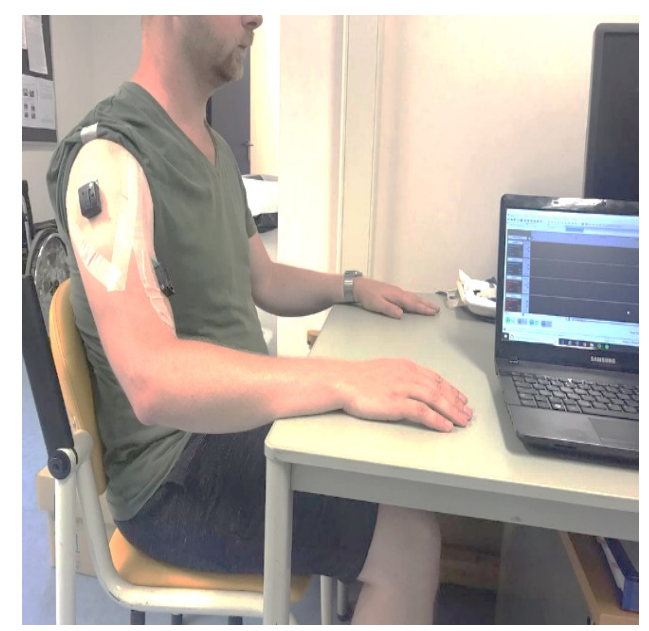

Figure 1. Participant with (A) Kinesio tape inhibitory muscle technique application and (B) non-elastic (microporous) tape application in the same configuration, both with the Delsys EMG sensors on biceps brachii and deltoid 


\section{HUMAN MOVEMENT}

R.S. Kós, T.W.J. Janssen, A. Cliquet Junior, Use of the inhibitory Kinesio taping

provide a good overview of the upper limb function through specific limb changes among individuals with upper limb commitment, with 19 items related to the shoulder, elbow, and wrist function. The 19 items are divided into 4 subscales, which are grasp, grip, pinch, and gross movement. The test scores are related to the performance and range from 0 to 3 , depending on the quality of the movement; in each item, the time in seconds to complete the task should also be obtained $[17,18]$. In this study, as there were only apparently healthy volunteers, the score related to the movement quality was not obtained, and the time in seconds to perform each task was used and compared between the tape conditions. ARAT was chosen for the healthy population because it uses specific materials like cylinders, cubes, and other objects for a standardized assessment of the arm function, being a proper tool for this evaluation, even in a population with any commitment.

\section{Statistical analysis}

Data were analysed with IBM SPSS 24 (IBM SPSS Statistics 24, SPSS Inc., Chicago, IL, USA). Exploratory data analysis was used for the characterization measures (frequency, percentage, mean, and standard deviation). The data had a normal distribution according to the Shapiro-Wilk test, and one-way ANOVA for repeated measures was performed. The level of significance was 5\%. The EMG peak results of the 3 trials in milliseconds of each muscle and the values in seconds of the ARAT items were used for analysis and comparison. Since there was only one group of ablebodied participants, the within-subject factor, responsible for detecting differences within the group related to the intervention, showed which tape condition had or had not enhanced the scores.

\section{Ethical approval}

The research related to human use has complied with all the relevant national regulations and institutional policies, has followed the tenets of the Declaration of Helsinki, and has been approved by the Scientific and Ethical Review Board of the Faculty of Behavioural and Movement Sciences of the Vrije Universiteit Amsterdam.

\section{Informed consent}

Informed consent has been obtained from all individuals included in this study.

\section{Results}

The 20 apparently healthy participants had a mean age of $31.8 \pm 8.5$ years (Table 1 ).

The one-way ANOVA for repeated measures revealed no significant differences in the EMG muscle activity among the 3 different techniques. The data were analysed from the tasks in each isolated muscle. The tape conditions and trials within the same task were compared, and the final comparison had the objective to find any statistically significant differences, crossing the tape condition data with the trials (Table 2).

In the analysis of ARAT, there were statistically significant differences in time scores and in the sum of all dimensions (total) $(p=0.04)$, and in the dimension of grip ( $p=0.006)$. The other dimensions (grasp, pinch, and gross motor) did not show statistically significant differences between the tape conditions.

The mean and standard deviation of both dimensions that presented significant differences can suggest that KT could have influenced these differences, on the basis of the shorter time of execution when compared with the other dimensions (Table 3).

Table 1. Characteristics of the sample

\begin{tabular}{lccl}
\hline Sex & $n$ & Age (years) & \multicolumn{1}{c}{ Dominance } \\
\hline Female & 7 & $34.28 \pm 11.68$ & $100 \% \mathrm{RH}$ \\
Male & 13 & $30.53 \pm 6.03$ & $\begin{array}{l}84.61 \% \mathrm{RH}(n=11) \\
\end{array}$ \\
& & $15.38 \% \mathrm{LH}(n=2)$ \\
Total & 20 & \multirow{2}{*}{$31.85 \pm 8.33$} & $90 \% \mathrm{RH}(n=18)$ \\
& & & $10 \% \mathrm{LH}(n=2)$ \\
\hline
\end{tabular}

RH - right-handed, LH - left-handed

Table 2. Values of $p$ from electromyography through ANOVA by task

\begin{tabular}{lcccccc}
\hline Factor & Bi T1 & Bi T2 & Bi T3 & De T1 & De T2 & De T3 \\
\hline Tape conditions & 0.57 & 0.38 & 0.79 & 0.41 & 0.19 & 0.21 \\
Trials & 0.99 & 0.70 & 0.21 & 0.33 & 0.11 & 0.41 \\
Conditions and trials & 0.32 & 0.12 & 0.76 & 0.95 & 0.89 & 0.72 \\
\hline
\end{tabular}

Bi - biceps, De - deltoid, T1 - task 1 (reach the top of the head), T2 - task 2 (reach the mouth), T3 - task 3 (throw the ball) 
Table 3. Mean and standard deviation values and $p$-values for the ARAT dimensions and total

\begin{tabular}{lrrrl}
\hline Dimension & \multicolumn{1}{c}{ No tape } & Kinesio tape & Non-elastic tape & $p$ \\
\hline Grasp (s) & $15.26 \pm 2.20$ & $14.88 \pm 2.62$ & $15.63 \pm 2.56$ & 0.08 \\
Grip (s) & $14.73 \pm 3.14$ & $13.77 \pm 2.55$ & $14.30 \pm 2.45$ & 0.006 \\
Pinch (s) & $16.66 \pm 2.88$ & $16.36 \pm 3.14$ & $16.54 \pm 2.53$ & 0.47 \\
Gross motor (s) & $6.62 \pm 0.89$ & $6.52 \pm 0.82$ & $6.69 \pm 0.90$ & 0.37 \\
Total (s) & $53.28 \pm 8.06$ & $51.55 \pm 8.66$ & $53.17 \pm 7.78$ & 0.04 \\
\hline
\end{tabular}

\section{Discussion}

The aim of this study was to analyse the effects of the inhibitory muscle technique of the KT method on the healthy muscle, in search of a trustworthy rehabilitation tool. Studies have shown statistically significant clinical results in function. However, the exact mechanism of the tape action is still unknown, with the hypotheses indicating that muscle facilitation occurs, the tape applied to the skin provides local stimulation through the tension of the elastic material, and the continuous stimulation during the period of application sends afferent information from the skin to the motor cortex, suggesting an enhancement in movement and performance [3]. Bravi et al. [11] also imply that the KT method increases the sensory inputs and influences the proprioception by the stimulation of the cutaneous mechanoceptors, induced by the pressure and stretching effect on the skin caused by the tape application.

The present study revealed that the KT inhibitory technique decreased the execution time of ARAT tasks when compared with the use of an NE placebo tape and no tape. Among all items scored in the grip dimension, there are items such as pouring water from one cup to another, fitting differently sized narrow tubes into cylinders, and moving a rounded wire over a cylinder. Decreasing the execution time of a task may suggest that the muscle inhibition mechanism proposed by the inhibitory technique when applied to a healthy muscle may adjust its physical condition, acting in the contraction-relaxation cycle, promoting fluidity, and spontaneously increasing the speed of the execution.

Poon et al. [9] discuss the idea of placebo when affirming that any structure attached to the skin can provide sensory input, in which a tension-free KT is not effective as a placebo application. Thus, a sham tape may not be considered a placebo as well. In this study, the microporous tape in the same configuration as KT was used to identify any difference between the 2 tape conditions, nullifying the idea that anything attached to the skin would stimulate it. However, the 2 different tapes did not differ from the tape-free condition in EMG activity, suggesting that the placebo could be used with both KT without tension and sham tape since both demonstrated differences as compared with the tapefree results.

In the present study, there were no significant differences in the KT inhibitory technique in healthy muscles, either in EMG activity or in upper limb function. In turn, Huang et al. [8] observed differences in EMG activity and no difference in function. Their study revealed that the muscle activity was increased, probably reducing components of overactivity and making the muscle more prepared to respond to a voluntary contraction, but the function presented no increase under the influence of KT. The authors used lower extremity muscle groups and jumping activity, differently from the activities proposed by this study, with arms against the gravity and open kinetic chain exercises. Such a comparison between groups may suggest that the KT method only works with high demands on muscle activity. However, Briem et al. [19] found that when the ankle received a sudden inversion perturbation, KT was not able to change the muscle activity in 43 male athletes, which discards the hypothesis of working on high muscle demands.

There are some limitations to the present study which must be taken into account when interpreting the results. The rigid tape used to compose a part of this intervention would, at times, limit all the assessed tasks, as well as the ARAT scores. Huang et al. [8] utilized a false application of KT in their study, obtaining favourable results when comparing this application with that following the principles of the KT method, a model also proposed by Poon et al. [9]. However, the findings in this study showed that the NE tape did not decrease the variables related to the movement when comparing the data with those for no tape or for KT, which, owing to the elastic property, does not limit the function. Future studies with similar objectives should consider a false KT application for comparison, in addition to proposing the same intervention format for groups with pain, spasticity, or overuse, checking the possible effects of the inhibitory muscle technique in other populations. 
The differences between the tape types were not sufficient to indicate the inhibitory technique as an efficient tool for enhancing the motor function and upper limb muscle activity in a healthy population. The comparison of all the variables analysed revealed a difference in the execution time related to KT in only one dimension and therefore in the total of the 4 dimensions assessed by ARAT, and no modification was observed in EMG activity.

\section{Conclusions}

According to the findings in this study, the KT inhibitory muscle technique and tape conditions did not evoke significant effects on muscle activity or arm function in apparently healthy muscles. Both KT and the placebo NE tape provided the same results when compared with the task performed with no tape.

There could be a tendency of conducting the movements faster with KT, related only to grip functions, as observed in the present study, but more research is needed to attest this influence.

\section{Acknowledgements}

This study was financed in part by the Coordenação de Aperfeiçoamento de Pessoal de Nível Superior (CAPES), Brazil (Finance Code: 001).

\section{Disclosure statement}

No author has any financial interest or received any financial benefit from this research.

\section{Conflict of interest}

The authors state no conflict of interest.

\section{References}

1. Lemos TV, Pereira KC, Protássio CC, Lucas LB, Matheus JPC. The effect of Kinesio Taping on handgrip strength. J Phys Ther Sci. 2015;27(3):567-570; doi: 10.1589/jpts.27.567.

2. Yang J-M, Lee J-H. Is Kinesio taping to generate skin convolutions effective for increasing local blood circulation? Med Sci Monit. 2018;24:288-293; doi: 10.12659/ MSM.905708.

3. Cai C, Au IPH, An W, Cheung RTH. Facilitatory and inhibitory effects of Kinesio tape: fact or fad? J Sci Med Sport. 2016;19(2):109-112; doi: 10.1016/j.jsams.2015. 01.010 .

4. Kim K-M, Davis B, Hertel J, Hart J. Effects of Kinesio taping in patients with quadriceps inhibition: a randomized, single-blinded study. Phys Ther Sport. 2017; 24:67-73; doi: 10.1016/j.ptsp.2016.08.015.
5. Kase K, Lemos TV, Dias EM. Kinesio Taping - introduction to the method and muscle techniques [in Portuguese]. São Paulo: Andreoli; 2013.

6. Kim JY, Kim SY. Effects of Kinesio tape compared with non-elastic tape on hand grip strength. J Phys Ther Sci. 2016;28(5):1565-1568; doi: 10.1589/jpts.28.1565.

7. Jung H-J, Lee J-Y, Hwang J-K, Choi B-R. Comparison of efficiency of elastic and non-elastic taping on induced quadriceps fatigue by knee extension exercise. J Phys Ther Sci. 2017;29(12):2199-2200; doi: 10.1589/jpts. 29.2199.

8. Huang C-Y, Hsieh T-H, Lu S-C, Su F-C. Effect of the Kinesio tape to muscle activity and vertical jump performance in healthy inactive people. Biomed Eng Online. 2011;10:70; doi: 10.1186/1475-925X-10-70.

9. Poon KY, Li SM, Rooper MG, Wong MKM, Wong O, Cheung RTH. Kinesiology tape does not facilitate muscle performance: a deceptive controlled trial. Man Ther. 2015;20(1):130-133; doi: 10.1016/j.math.2014. 07.013.

10. Kim W-I, Choi Y-K, Lee J-H, Park Y-H. The effect of muscle facilitation using Kinesio taping on walking and balance of stroke patients. J Phys Ther Sci. 2014;26(11): 1831-1834; doi: 10.1589/jpts.26.1831.

11. Bravi R, Quarta E, Cohen EJ, Gottard A, Minciacchi D. A little elastic for a better performance: Kinesiotaping of the motor effector modulates neural mechanisms for rhythmic movements. Front Syst Neurosci. 2014;8:181; doi: 10.3389/fnsys.2014.00181.

12. Dos Santos Vieira PC, Almeida Oliveira R, Fernandes LM, Pereira K, Pasucci Sande de Souza LA. Augmented muscle activation in reaching movements after stroke. Hum Mov. 2020;21(3):39-46; doi: 10.5114/hm.2020. 91344.

13. Garcia T, Dal Pupo J, Knihs DA, Furlaneto Rodrigues OA, Zimmermann HB, Padulo J. Single- versus multi-joint isometric protocols to induce a post-activation potentiation effect on squat jump performance. Hum Mov. 2020;21(3):71-80; doi: 10.5114/hm.2020.91348.

14. Hermens HJ, Freriks B, Merletti R, Stegeman D, Blok J, Rau G, et al. European recommendations for surface electromyography: results of the SENIAM project. Roessingh Research and Development; 1999.

15. Gates DH, Walters LS, Cowley J, Wilken JM, Resnik L. Range of motion requirements for upper-limb activities of daily living. Am J Occup Ther. 2016;70(1):7001350 010p1-7001350010p10; doi: 10.5014/ajot.2016.015487.

16. Davidson J, Pinheiro Tsopanoglou S, Zuniga Dourado V, Carvas N Jr, Miyashiro Nunes Dos Santos A, Goulart AL, et al. Differences in activation of the vastus lateralis muscle during maximal exercise test between very-lowbirth-weight preterm and full-term children. Hum Mov. 2020;21(3):31-38; doi: 10.5114/hm.2020.91343.

17. Santanielo N, Nóbrega SR, Scarpelli MC, Alvarez IF, Otoboni GB, Pintanel L, et al. Effect of resistance training to muscle failure vs non-failure on strength, hypertrophy and muscle architecture in trained individuals. 
Biol Sport. 2020;37(4):333-341; doi: 10.5114/biolsport. 2020.96317.

18. Yozbatiran N, Der-Yeghiaian L, Cramer SC. A standardized approach to performing the Action Research Arm Test. Neurorehabil Neural Repair. 2008;22(1):7890; doi: 10.1177/1545968307305353.

19. Briem K, Eythörsdöttir H, Magnúsdóttir RG, Pálmarsson R, Rúnarsdöttir T, Sveinsson T. Effects of Kinesio Tape compared with nonelastic sports tape and the untaped ankle during a sudden inversion perturbation in male athletes. J Orthop Sports Phys Ther. 2011;41(5): 328-335; doi: 10.2519/jospt.2011.3501. 